\title{
Properties of mid- to far-infrared dust emission in the nearby superwind galaxy M82
}

\author{
Ko Arimatsu ${ }^{1,2}$, Takashi Onaka ${ }^{1}$, Itsuki Sakon $^{1}$, Fumi Egusa ${ }^{2}$, \\ and Hidehiro Kaneda ${ }^{3}$ \\ ${ }^{1}$ Department of Astronomy, Graduate School of Science, The University of Tokyo, \\ 7-3-1 Hongo, Bunkyo-ku, Tokyo 113-0033, Japan. \\ email: arimatsu@astron.s.u-tokyo.ac.jp \\ ${ }^{2}$ Institute of Space and Aeronautical Science, Japan Aerospace Exploration Agency, \\ 3-1-1 Yoshinodai, Sagamihara, Kanagawa 229-8510, Japan. \\ email: arimatsu@ir.isas.jaxa.jp \\ ${ }^{3}$ Graduate School of science, Nagoya University, \\ Chikusa-ku, Nagoya 464-8602, Japan.
}

\begin{abstract}
Using the reconstructed imaging data obtained with the Infrared Camera (IRC) on board AKARI, mid-infrared (MIR; 5-30 $\mu \mathrm{m}$ ) emission characteristics of the superwind galaxy M82 are studied. The MIR images at four wavelengths $(7,11,15$, and $24 \mu \mathrm{m})$ show extended (out to distances of $4 \mathrm{kpc}$ ) emission mainly from polycyclic aromatic hydrocarbons (PAHs). The MIR SED of M82 halo is surprisingly constant. Using far-infrared imaging data obtained by Herschel/SPIRE, we reveal that the PAH abundance relative to the big (sub-micron sized) grains radially increases by about a factor of three. These results imply that PAHs may be formed in small and dense molecular clumps in the halo and efficiently supplied to the intergalactic space by the galactic superwind.
\end{abstract}

Keywords. infrared: galaxies, galaxies: starburst

\section{Introduction}

Galactic superwinds carry neutral and molecular gas and dust from the galactic disk into the halo and the intergalactic space (e.g. Heckman et al. 1990). It is valuable to investigate the mid- to far-infrared dust emission properties in the superwind to understand the chemical enrichment process of the galactic halo and the intergalactic space.

M82 (NGC 3034) is one of the most studied superwind galaxies at various wavelengths, thanks to its proximity and edge on geometry. Engelbracht et al. (2006) and Kaneda et al. (2010) found very extended mid- to far-infrared emission associated with the superwind and the tidal arms in the halo region of M82. The observed mid-infrared (MIR) emission in the halo is thought to come from PAHs, which survive in the harsh superwind environment. Further investigations are required to understand the properties of the extended infrared emission.

This report presents the latest results of the re-analysis of the imaging data obtained with $A K A R I /$ IRC, which is provided by artifact-subtraction processes and a new image reconstruction method developed by Arimatsu et al. (2011). The latest results of the comparison between the AKARI MIR data and the far-infrared (FIR) data obtained with Herschel/SPIRE (Roussel et al. 2010) are also presented in this report. 

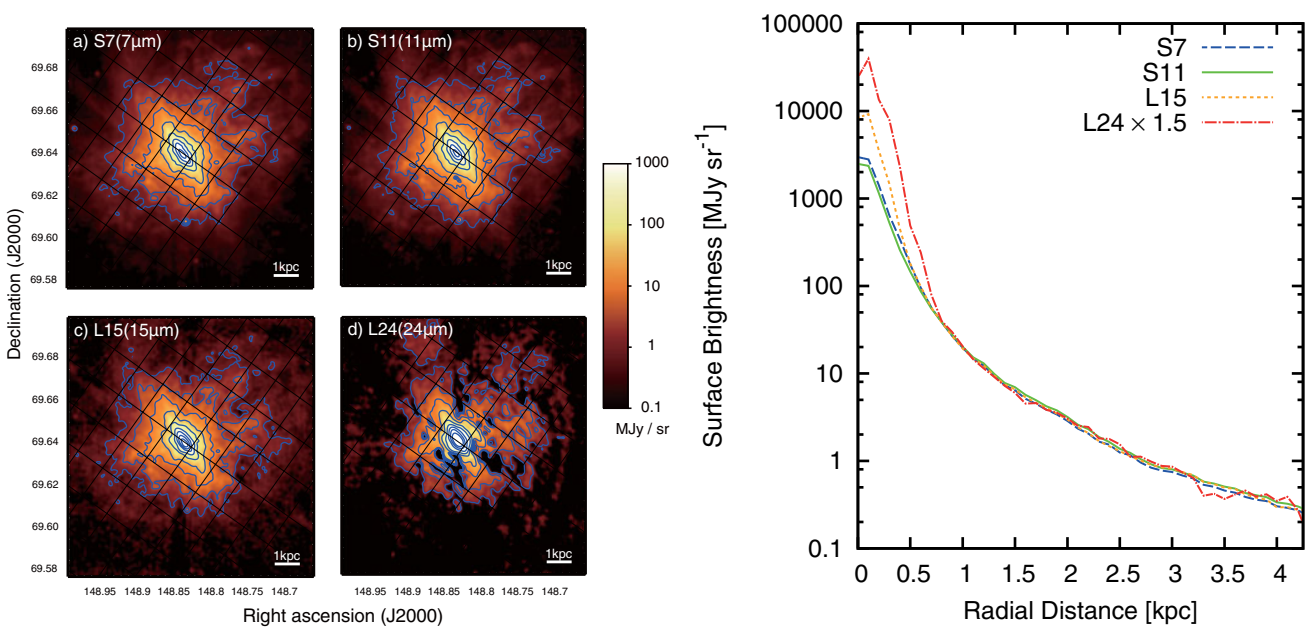

Figure 1. Left: IRC MIR band images of M82. Right: Radial surface brightness properties.
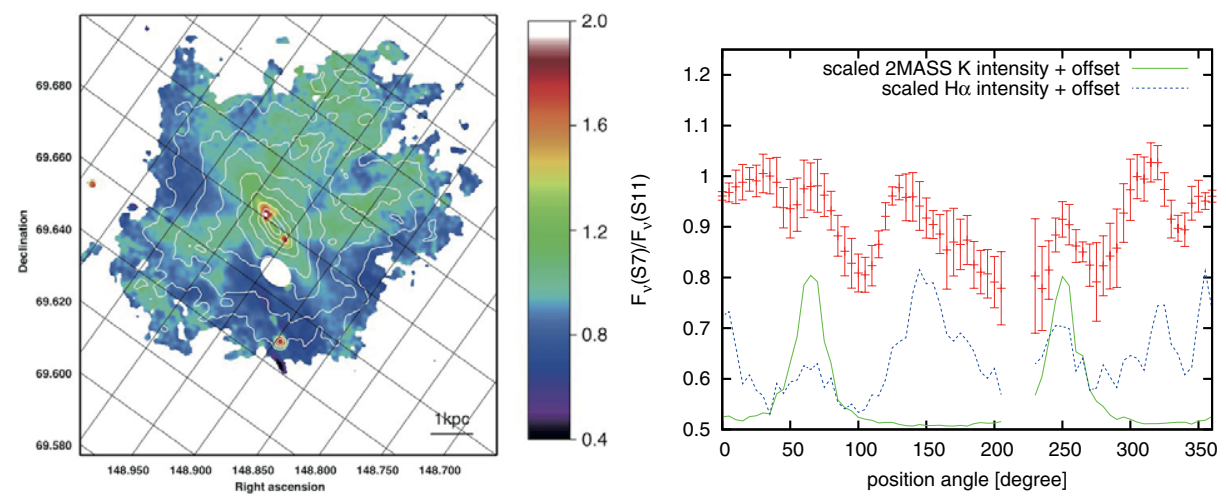

Figure 2. Left: $7 \mu \mathrm{m} / 11 \mu \mathrm{m}$ flux ratio map of M82. Right: Position angle profile of $7 \mu \mathrm{m} / 11 \mu \mathrm{m}$ flux ratio overlaid with the $\mathrm{H} \alpha$ and 2 MASS K band intensity profiles.

\section{Mid- to far-IR Emission from M82}

The reconstructed four band $(7,11,15$, and $24 \mu \mathrm{m})$ MIR images of M82 taken with the $A K A R I /$ IRC MIR channels, which are shown in the left panel of Figure 1, reveal extended emission in the halo. The spatial distribution of the emission is surprisingly similar at the four wavelenths.

MIR radial profile. Fig. 1 (right-hand panel) shows the radial surface brightness profiles of M82 at the IRC four MIR bands. The MIR radial surface brightness profiles are surprisingly similar among the four bands at $r>1 \mathrm{kpc}$. These profiles can be well approximated by a power law function. The fitted power-law index is about -3 , which indicates that the intensity density of the PAH emission, which reflects the PAH density distribution and the intensity of the radiation field, gently decreases with radius $\left(\propto r^{-4}\right)$.

MIR intensity ratio map. The $7 \mu \mathrm{m} / 11 \mu \mathrm{m}$ flux ratio is thought to trace the ratio between the 7-9 $\mu \mathrm{m}$ PAH features (enhanced when PAHs are ionized) and the PAH and very small grain emission at $10-13 \mu \mathrm{m}$ (Sakon et al. 2006). The $7 \mu \mathrm{m} / 11 \mu \mathrm{m}$ flux ratio map (Figure 2 left) shows a relatively flat flux ratio at $r>1 \mathrm{kpc}$. This result suggests that the ionization fraction of PAHs is independent of galactocentric radius. $7 \mu \mathrm{m} / 11 \mu \mathrm{m}$ position angle profile (Fig. 2 right-hand panel) shows a trend that seems to correlate 


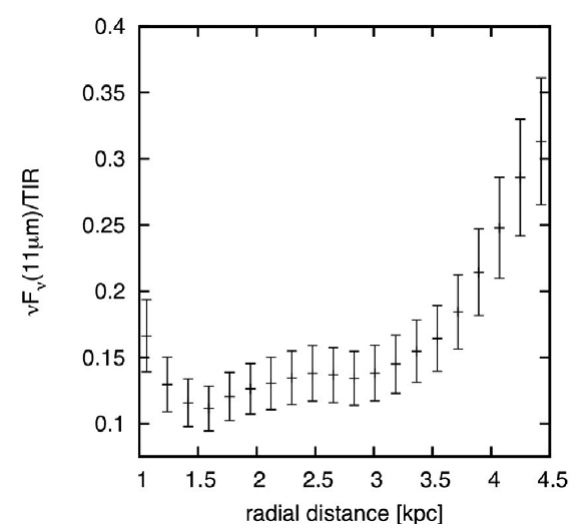

Figure 3. Radial profile of $11 \mu \mathrm{m} / \mathrm{TFIR}$.

with the $\mathrm{H} \alpha$ intensity profile. This colour variation suggests that PAHs are ionized near the $\mathrm{H} \alpha$ emission, with neutral PAHs located behind the ionized gas regions (which may be associated with molecular gas - e.g., Walter et al. 2002).

PAH abundance relative to the FIR emitting dust. To compare the PAH abundance relative to bigger, classical dust grains, we use the Herschel/SPIRE photometric data and estimate the mass and temperature of bigger grains to compare with the MIR data. The radial profile of the intensity ratio between the $11 \mu \mathrm{m}$ intensity and the total FIR luminosity is shown in Figure 3. This result is interpreted as a radial increase of PAHs relative to big grains.

The explanation of this trend is not yet clear. This result may suggest that PAHs are transported into the halo more efficiently than big grains. This abundance trend could also be due to the formation of PAHs in the dense $\left(n_{H} \sim 10^{4} \mathrm{~cm}^{-3}\right)$ molecular clumps in the superwind (Herbst 1991, Greenberg et al. 2000). However, the formation efficiency is insufficient by more than three orders of magnitude to account for the trend. Alternatively, this trend could be explained by the formation of PAHs by shock-induced shattering of big grains (Jones et al. 1996) in the turbulent superwind environment. In any case, PAHs have to be shielded from hot $\left(\sim 10^{6} \mathrm{~K}\right)$ plasma gas, which destroys PAHs by electron sputtering (Micelotta et al. 2010). The radial increase of the PAH abundance relative to big grains is thus suggested to be due to the enhancement of the emergence of PAHs from the molecular clumps in the halo, which can be a container and may be the source of the delicate materials in the harsh environment. Wider area $(5 \mathrm{kpc} \times 5$ $\mathrm{kpc}$ ), and multiwavelength ( $\mathrm{CO}, \mathrm{IR} \mathrm{H}_{2}$ lines, etc.) observations are required to constrain a clear explanation of this result.

\section{References}

Arimatsu, K., et al. 2011, PASP, 123, 981

Engelbracht, C. W., Kundurthy, P., Gordon, K. D., et al. 2006, ApJ, 642, L127

Heckman, T. M., Armus, L., \& Miley, G. K. 1990, ApJS, 74, 833

Herbst, E. 1991, ApJ, 366, 133

Jones, A. P., Tielens, A. G. G. M., \& Hollenbach, D. J. 1996, ApJ, 469, 740

Kaneda, H., Ishihara, D., Suzuki, T., et al. 2010, A\&A A, 514, 14

Micelotta, E. R., Jones, A. P., \& Tielens, A. G. G. M. 2010b, A\&AA, 510, A37

Roussel, H., et al. 2010, A\&GA, 518, L66

Sakon, I., et al. 2006, ApJ, 651, 174

Walter, F., Weiss, A., \& Scoville, N. 2002, ApJ, 580, L21 